\title{
ESTUDO DO SOLO-CIMENTO-AUTOADENSÁVEL PRODUZIDO COM SOLOS DA REGIÃO DO PORTO-PT. PARTE II: AVALIAÇÃO DA RESISTÊNCIA E DESEMPENHO MECÂNICO POR MEIO DA MEDIÇÃO DA RESISTIVIDADE ELÉTRICA E VELOCIDADE DE PROPAGAÇÃO DE ONDAS DE ULTRASSOM
}

\section{Study of the self-compacting soil-cement with soils from the Porto-Pt. Part II: Evaluation of resistance and mechanical performance by measuring the electrical resistivity and velocity of propagation of ultrasound wave}

\author{
Marco Antônio de Morais Alcantara ${ }^{1}$ Sandra Conceição Barbosa Nunes ${ }^{2}$, \\ João Filipe Meneses Espinheira Rio ${ }^{3}$
}

Recebido em 16 de maio de 2014; recebido para revisão em 11 de julho de 2014; aceito em 26 de agosto de 2014; disponivel on-line em 06 de setembro de 2014.

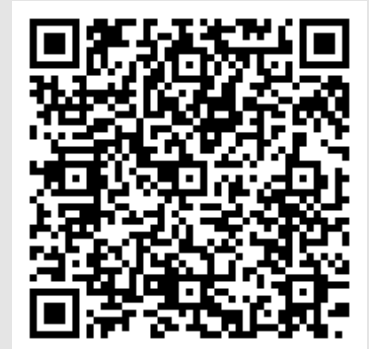

PALAVRAS CHAVE:

Solo-cimento;

Autoadensável;

Reologia;

Compacidade;

\section{KEYWORDS:}

Soil-cement;

Self-compacting,

Rheology;

Compactness.

\begin{abstract}
RESUMO: O artigo apresenta um estudo do solo-cimento autoadensável, sobre utilização da medição das propriedades relativas à resistividade elétrica e da velocidade de propagação de ondas de ultrassom, como meios de se buscar subsídios para esclarecer o processo de estabilização, bem como de se encontrar um meio indireto para a estimativa do valor da resistência e do desempenho mecânico. Para tanto, foi realizado um experimento a partir da utilização de um solo residual da região do Porto-Pt, cimento Portland, água, e aditivo de atuação estérica e eletrostática. A proporção dos materiais foi adotada a partir do peso seco do solo, conforme teores que variaram de 20 , 25 , e $30 \%$ de cimento, 41, 44 e $47 \%$ para a água, e 0,66, 0,80 e 0,93\% para a variação do teor do superplastificante. Os ensaios no realizados no estado fresco procuraram seguir os protocolos de avaliação utilizados para os casos de argamassas e de concretos autoadensáveis; e, no estado endurecido, foram realizados os ensaios de resistência à compressão simples, resistência à flexão, e a avaliação dos valores da resistividade elétrica e da velocidade da propagação de ondas ultrassom. Os resultados indicaram haver aproximação entre o solo-cimento-auto-adensável e os concretos e argamassas; bem como, a variação dos valores da resistividade elétrica e da velocidade de propagação de ondas de ultrassom se apresentaram coerentes com a idade de cura e com os valores de resistência mecânica alcançados.
\end{abstract}

ABSTRACT: The article presents a study of the soil-cement self-compacting, on the feasibility of using the measurement of the properties on the electrical resistivity and the speed of propagation of ultrasound waves as a means of seeking information to clarify the stabilization process as well as to find an indirect means for estimating the value of resistance and mechanical performance. Therefore, an experiment was carried from the use of a residual soil of the region of Porto-Pt, Portland cement, water and additive steric and electrostatic actuation. The ratio of materials was taken from the dry weight of soil as levels varying from 20,25 , and $30 \%$ cement, 36,39 and $42 \%$ for water, and 4,6 and $8 \%$ for changes the content of superplasticizer. The tests performed on fresh state sought to follow the evaluation protocols used for cases of mortars and self-compating concretes; in the hardened state, the tests were performed compressive strength, flexural strength, and evaluation of the values of electrical resistivity and the speed of propagation of ultrasound waves. The results showed approach between the soil-cement-and self-compacting concrete and mortar; strength values correlated with the values of the water / cement ratio; well as the variation of the values of electrical resistivity and the propagation velocity of ultrasonic waves is presented consistent with age curing and mechanical resistance values achieved.

\footnotetext{
* Contato com os autores:

${ }^{1}$ e-mail :alcantar@dec.feis.unesp.br (M. A. de M. Alcantara)

Professor Assistente, Doutor, Departamento de Engenharia Civil - Faculdade de Engenharia de llha Solteira (FEIS/UNESP).

2e-mail : snunes@fe.up.pt ( S. C. B. Nunes )

Professora Auxiliar, Doutora, Departamento de Engenharia Civil - Faculdade de Engenharia da Universidade do Porto (FEUP).

${ }^{3}$ e-mail : joao.rio@fe.up.pt (J.F.M. E. Rio )

Investigador Auxiliar, Doutor, Departamento de Engenharia Civil - Faculdade de Engenharia da Universidade do Porto (FEUP).
} 


\section{INTRODUÇÃO}

Segantini e Alcantara (2010) apresentam o solo-cimento plástico, concebido de modo a ser compactado sob a ação do seu peso próprio, utilizado particularmente para a produção de estacas moldadas "in loco", e cujos requisitos de desempenho estão relacionados às exigências geotécnicas para fundações. Os autores consideram para este tipo de material aspectos de consistência e de resistência mecânica em função do teor de água incorporada. Neste sentido misturas solocimento são compreendidas como mistura íntima entre os constituintes: solo, cimento, e água, sendo estas misturas alcançadas por meio de misturadores mecânicos utilizados na construção civil.

Com base nas possíveis propriedades de auto adensabilidade das misturas de solo-cimento, fluidificadas por meio do auxílio de aditivos superplastificantes, Berté e Alcantara (2013) apresentam um estudo visando a auto adensabilidade do solo-cimento, de maneira que este possa ser aplicado para a fabricação de elementos diversos quanto à forma, atendendo a diferentes classes de resistência mecânica, e devendo atender também às exigências que favoreçam a durabilidade. Este tem como vantagem dispensar o uso de equipamentos de prensagem, e requer a utilização de equipamentos básicos para a realização da mistura, visando atender à produção de componentes para a construção civil.

Considera-se importante estabelecer uma diferenciação entre o solo-cimento tradicional, compactado, e o solo-cimento na forma autoadensável, sobretudo em termos dos parâmetros de dosagem e dos aspectos tecnológicos. Enquanto o solo-cimento tradicional se enquadra no escopo da geotecnia, obedecendo a critérios conforme apresentados em Consoli et al. (2007), e, também, Fonseca et al. (2009), o solocimento autodensável busca relações próximas à dos concretos e argamassas, conforme apresentados em BERTÉ e ALCANTARA (2013). Dentro deste contexto, as reações de hidratação do cimento desempenham um papel relevante, sobretudo pela formação de compostos cimentantes, silicatos e aluminatos de cálcio hidratados e cristalizados.

A resistividade dos materiais pode estar associada à porosidade dos materiais. Para os casos dos concretos, estes podem transportar cargas elétricas, sendo esta de natureza eletrolítica, viabilizada pelos íons dissolvidos na água que preenche de maneira total ou parcial a rede de poros interconectados da pasta. Como fatores para a variação deste tipo de corrente elétrica podem ser apontados a natureza da estrutura porosa, a distribuição da água, a temperatura e sobretudo da concentração iônica na solução intersticial (ANDRADE, 1992). Segundo Rosa (2005), a resistividade pode ser aumentada com o grau de hidratação e com a utilização de adições ativas, onde, este comportamento está associado à uma menor condução elétrica específica na solução dos poros, e a um refinamento dos poros.

Por outro lado, as ondas sonoras necessitam de um meio material para propagarem, e a sua transmissão pode sofrer a influência das propriedades intrínsecas do material, como a sua massa, a densidade, e o tipo de forças de coesão. Neste sentido, podem-se associar as condições de transmissão a um tipo de material. Além da velocidade de propagação do som, são relevantes as reflexões, e o eco, causados pela presença de obstáculos, e de descontinuidades (MALHOTRA e CARINO, 2004). Neste sentido, a utilização da propagação de ondas sonoras pode ser utilizada com meio de se compreender sobre a microestrutura de um material, e de avaliar algumas de suas propriedades.

Considerando que o solo-cimento autoadensável se processa de modo similar aos dos concretos e argamassas quanto aos seus mecanismos de estabilização, com a formação de compostos cimentantes oriundos da estabilização do cimento Portland, considerou-se que as propriedades relativas à resistividade elétrica e da velocidade de propagação de ondas de ultrassom, poderão guardar relações com a evolução do processo de estabilização, bem como, de se encontrar correspondências entre os valores da resistência e do desempenho mecânico. 


\section{OBJETIVO}

Os objetivos deste trabalho foram de se utilizar a medição das propriedades relativas à resistividade elétrica e da velocidade de propagação de ondas de ultrassom como meios de se buscar subsídios para esclarecer o processo de estabilização do solo-cimento autoadensável, bem como de se encontrar um método indireto para a estimativa do valor da resistência e do desempenho mecânico, e ainda, de se poder estabelecer previsão do valor da resistência mecânica nos primeiros dias, como ensaios não destrutivos.

\section{MATERIAIS E MÉTODOS}

Para o desenvolvimento do trabalho foram utilizados os seguintes materiais: solo residual saprolítico da região de Porto-Pt, cimento Portland do tipo cimento CEM I 42.5, superplastificante de ação estérica e eletrostática, e água.

A metodologia em termos do seu plano de ensaio buscou que se explorasse a variação dos agentes fluidificantes, dados pela água e pelo superplasticante, a as condições de viscosidade, dados pelo cimento e o solo, de modo a resultar nas composições que são apresentadas no Quadro 1. As misturas para esse estudo são apresentadas no Quadro 1.

As misturas eram produzidas em argamassadeira. Uma vez que estas eram concluídas, se procedia a realização dos ensaios de validação no estado fresco: (1) os ensaios de espalhamento em escoamento livre, "slump-flow", conforme Nunes (2001), e (2) os ensaios de escoamento confinado, através do ensaio do funil, conforme NUNES (2001) e ALCANTARA (2004). Feitos os ensaios do estado fresco, procedia-se a moldagem, vertendo o conteúdo da cuba do misturador nos moldes específicos, sem o auxílio de vibração ou de qualquer outro esforço mecânico, contando exclusivamente com a energia dada pelo peso próprio do material. Como moldes se utilizou de fôrmas permitindo a fabricação de 3 corpos de prova prismáticos de $4 \times 4 \times 6 \mathrm{~cm}$, e moldes cilíndricos com diâmetro de $46 \mathrm{~mm}$ e altura de $31 \mathrm{~mm}$. Para cada tipo de caso Foram adotadas 3 unidades experimentais, sejam, 3 moldes prismáticose 3 moldes cilíndricos. Os procedimentos para a amassadura e para a realização dos ensaios no estado fresco são descritos na Parte 1 deste trabalho "Estudo do Solo-Cimento Autoadensável com Solos da Região do Porto-Pt", sobre a caracterização de propriedades mecânicas.

\begin{tabular}{|c|c|c|c|c|c|c|c|c|}
\hline \multicolumn{7}{|c|}{ Caso do superplastificante/solo mantido fixo } & \multicolumn{2}{|c|}{$\begin{array}{c}\text { Caso do } \\
\text { superplastificante/solo } \\
\text { mantido Variável }\end{array}$} \\
\hline & & $\begin{array}{l}\text { Mistura 1- } \\
\text { referência }\end{array}$ & $\begin{array}{c}\text { Mistura } \\
2\end{array}$ & $\begin{array}{c}\text { Mistura } \\
3\end{array}$ & $\begin{array}{c}\text { Mistura } \\
\quad 4\end{array}$ & $\begin{array}{l}\text { Mistura } \\
5\end{array}$ & Mistura 6 & Mistura 7 \\
\hline \multicolumn{2}{|c|}{ Solo (g) } & 1508.33 & 1508.33 & 1508.33 & 1508.33 & 1508.33 & 1508.33 & 1508.33 \\
\hline \multicolumn{2}{|c|}{$\begin{array}{l}\text { Cimento CEM I } 42.5 \\
\text { R-Secil-Maceira (g) }\end{array}$} & 301.67 & 301.67 & 301.67 & 377.08 & 452.50 & 301.67 & 301.67 \\
\hline \multicolumn{2}{|c|}{ Água } & 663.67 & 708.92 & 618.42 & 663.67 & 663.67 & 663.67 & 663.67 \\
\hline \multicolumn{2}{|c|}{ Supeplastificante (V3008) (g) } & 12.07 & 12.07 & 12.07 & 12.07 & 12.07 & 10 & 14 \\
\hline \multicolumn{2}{|c|}{ Água 1 (g) } & 530.93 & 567.13 & 494.73 & 530.93 & 530.93 & 567.13 & 494.73 \\
\hline \multicolumn{2}{|c|}{ Água 2 (g) } & 132.73 & 141.78 & 123.68 & 132.73 & 132.73 & 141.78 & 123.68 \\
\hline \multirow{3}{*}{$\begin{array}{l}\text { Relações entre } \\
\text { os constituintes } \\
\text { das misturas }\end{array}$} & cimento/solo & \multicolumn{3}{|c|}{$20 \%$} & $25 \%$ & $30 \%$ & \multicolumn{2}{|c|}{$20 \%$} \\
\hline & agua/solo & $44 \%$ & $47 \%$ & $41 \%$ & --- & --- & \multicolumn{2}{|c|}{44} \\
\hline & sup/solo & $0,80 \%$ & $0,80 \%$ & $0,80 \%$ & $0,80 \%$ & $0,80 \%$ & $0,66 \%$ & $0,93 \%$ \\
\hline
\end{tabular}


As formas retangulares eram dotadas de pequenas telas metálicas dispostas nas extremidades, de modo a que as caldas vertidas as embebessem, e que estas então pudessem atuar como elétrodos acoplados às extremidades dos corpos de prova. Os corpos de prova eram após cobertos e dispostos por um período de 5 dias para que se pudesse efetuar a desmoldagem, de modo a não haver prejuízo para as redes e no cobrimento destas, viabilizando os ensaios posteriores de medição da resistividade elétrica e do ultrassom. Tantos os primeiros ensaios de resistividade elétrica dos corpos de prova assim como os de avaliação da velocidade de propagação de ondas de ultrassom eram realizados logo após a desmoldagem, sendo, a seguir, os corpos de prova encaminhados para a câmara úmida, seguindo-se pelo período de cura de 7, 14 e de 28 dias, sendo estes avaliados quanto ao valor da resistividade elétrica e da velocidade de propagação de ondas de ultrassom, e sujeitos aos ensaios de flexão simples e de compressão simples aos 28 dias de cura, em consonância com a norma NP EN 196 1, prescrita em IPQ (1996).

A avaliação da resistividade elétrica foi realizada com a aplicação de uma diferença de potencial nas extremidades dos corpos de prova, em elétrodos posicionados estrategicamente em faces opostas e planas, conforme o método de Werner, descrito em HOPE (2005). A representação esquemática do método é apresentada na Figura 1.

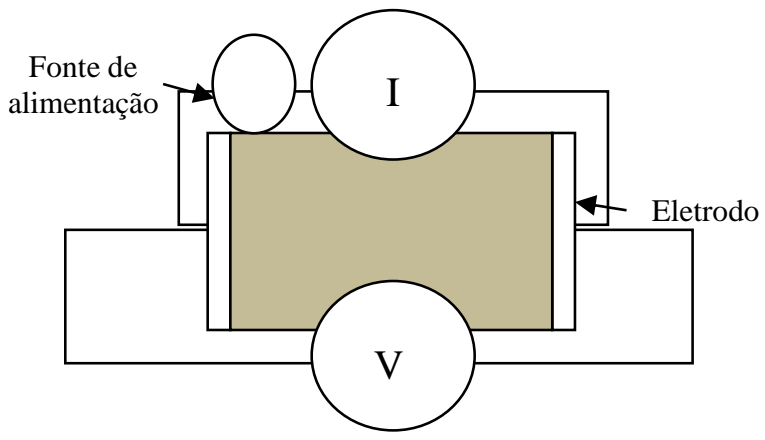

FIGURA 1: Representação esquemática do ensaio de avaliação de resistividade.

O valor da resistividade elétrica é então dado por meio da Equação 1:

$$
\rho=\frac{\text { V.A }}{\text { L.I }}
$$

Onde:

$\rho$ é o valor da resistividade ( $\Omega . m$ );

L é o comprimento do corpo de prova (m);

A é a área do elétrodo que está em contato com o corpo de prova $\left(\mathrm{m}^{2}\right)$;

V é a tensão (Volts);

I é a corrente (Ampere).

A Figura 2a ilustra os corpos de prova moldados, e dotados das telas metálicas que funcionam como elétrodos, e a Figura $2 b$ ilustra um caso da medição.
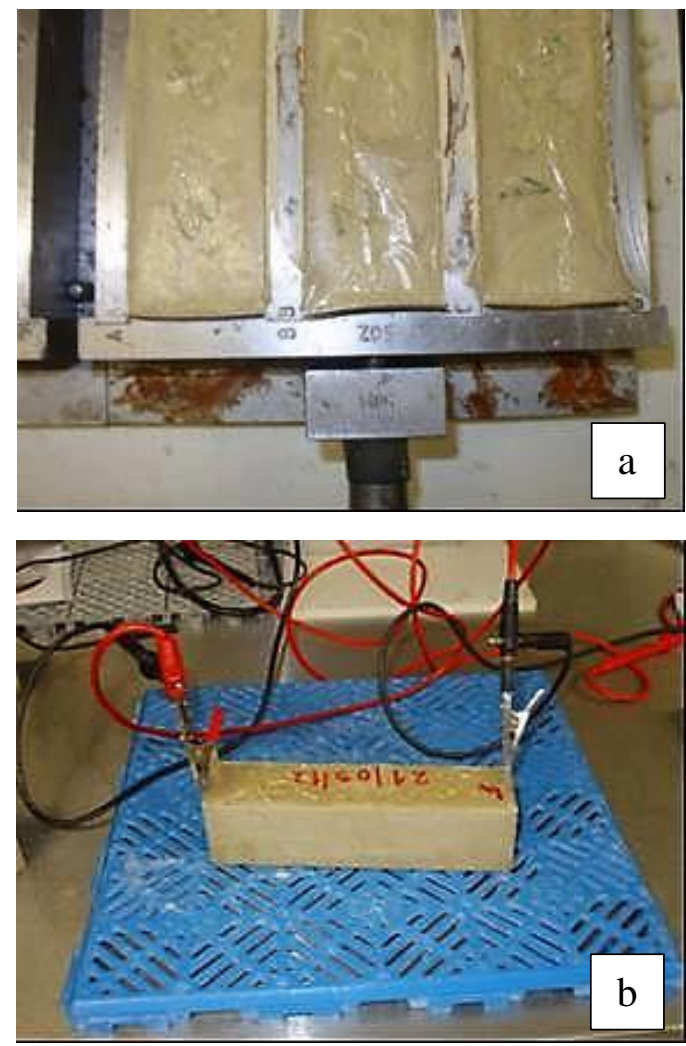

FIGURA 2: (a)Solo-cimento auto adensável aguardando a desmoldagem; (b) medição de resistividade por meio de elétrodos posicionados na amostra de solo-cimento auto adensável durante o período de cura.

Os ensaios de ultrassom são ensaios não destrutivos, utilizados para o concreto como descrito em MALHOTRA e CARINO (2004). Foi utilizado um equipamento que inclui 2 transdutores que funcionam como transmissor e receptor, geração de sinal, aquisição de sinal e computação do tempo de viagem, t. O sinal gerado é do tipo impulso ultra-sônico, sendo que o equipamento mede assim a velocidade de transmissão de impulsos ultra-sônicos, Ultrasonic Pulse Velocity, UPV. O equipamento foi utilizado para transmissão 
direta de ondas, de modo que, com os transdutores alinhados, se media a velocidade de propagação de ondas $\mathrm{P}, \mathrm{VP}$, também conhecidas como ondas principais, ondas de compressão ou ondas longitudinais. De maneira a se evitar possíveis interferências nos ensaios, a superfície do corpo de prova recebia sempre a aplicação de pintura de um material, normalmente do tipo glicerina ou graxa, que se interpunha entre esta superfície e o cabeçote. A Figura 3 ilustra o ensaio.

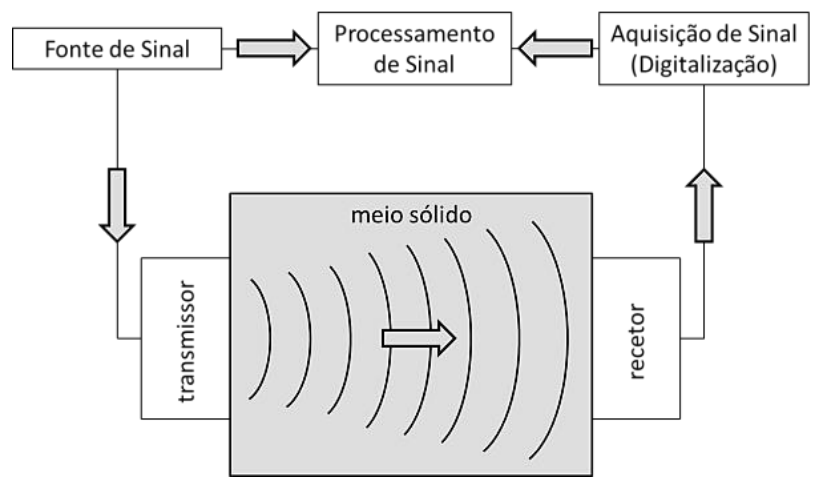

FIGURA 3: Esquema do solo-cimento auto-adensável em fase de cura submetido ao ensaio de ultra som.

Buscou-se resultados que pudessem ser utilizados de forma qualitativa, para comparar os resultados de diferentes corpos de prova de diferentes tipos, com diferentes misturas, amassaduras e idades. Sendo conhecida a distância percorrida, no caso dos corpos de prova seria o seu comprimento, $s=0,016 \mathrm{~m}$, pôde-se calcular a velocidade das ondas, de acordo com:

$$
\mathrm{V}_{\mathrm{p}}=\frac{\mathrm{s}}{\mathrm{t}}
$$

Onde:

$\mathbf{V}_{\mathrm{p}}$ é a velocidade de propagação das ondas $(\mathrm{m} / \mathrm{s})$;

$S$ é o comprimento do corpo de prova (m);

t é o tempo necessário para o percurso (s).

\section{RESULTADOS E DISCUSSÃO}

Com base nos valores apresentados no Quadro 1, o Quadro 2 apresenta os parâmetros das misturas estudadas.

Os resultados dos ensaios realizados para o material no estado fresco se encontram apresentados e discutidos na parte I deste trabalho, "Estudo do Solo-Cimento Autoadensável Produzidos com Solos da Região do Porto-Pt", relativo à caracterização de Propriedades mecânicas, onde, conforme relatado, as misturas se apresentaram em condições satisfatórias, atendendo aos requisitos para este tipo de material, sejam: fluidez, mobilidade e homogeneidade.

QUADRO 2: Parâmetros das misturas estudadas.

\begin{tabular}{|c|c|c|c|c|c|c|c|}
\hline & \multicolumn{7}{|c|}{ Mistura } \\
\hline Parâmetro & $\mathrm{M} 1$ & $\mathrm{M} 2$ & $\mathrm{M} 3$ & $\mathrm{M} 4$ & $\mathrm{M} 5$ & $\mathrm{M} 6$ & $\mathrm{M} 7$ \\
\hline a/c & 2,2000 & 2,3500 & 2,0500 & 1,7567 & 1,4667 & 2,2000 & 2,2000 \\
\hline a/(cim+solo) & 0,3667 & 0,3917 & 0,3417 & 0,3519 & 0,3385 & 0,3667 & 0,3667 \\
\hline a/solo & 0,4400 & 0,4700 & 0,4100 & 0,4400 & 0,4400 & 0,4400 & 0,4400 \\
\hline Sup/cim & 0,0400 & 0,0400 & 0,0400 & 0,0319 & 0,0267 & 0,0331 & 0,0464 \\
\hline Sup/solo & 0,0080 & 0,0080 & 0,0080 & 0,0080 & 0,0080 & 0,0066 & 0,0093 \\
\hline Cim/solo & 0,2000 & 0,2000 & 0,2000 & 0,2505 & 0,3000 & 0,2000 & 0,2000 \\
\hline
\end{tabular}

Mi: Mistura i 
O Quadro 3 apresenta os resultados dos ensaios mecânicos no estado endurecido, quando em ensaios de resistência à compressão simples, e em ensaios de flexão, respectivamente, distinguindo-se os valores médios, o desvio padrão e o coeficiente de variação, para as misturas do estudo, definidas conforme dos parâmetros das misturas.

Conforme o que foi apresentado sobre as propriedades mecânicas na Parte I deste estudo, os valores de resistência à flexão se apresentam correlacionados aos valores de resistência à compressão simples, de modo que, as análises possíveis entre os valores das propriedades mecânicas correlacionadas aos valores das propriedades de resistividade elétrica e de condutividade das ondas de ultrassom serão feitas com base nos valores da resistência à compressão simples. A Figura 4, a seguir, com base na superposição das curvas que puderam ser obtidas com base em cada tratamento descrito no item 3, procura associar os valores de resistência à compressão simples com os valores das relações água/cimento, calculadas a partir das informações de cada mistura

Pode-se observar que a variação do valor da resistência mecânica à compressão simples se dá de maneira inversamente proporcional ao valor da variação da relação água/cimento, de maneira análoga aos casos dos concretos e argamassas, e evidenciado na Parte I deste trabalho. O Quadro 4 apresenta os resultados da resistividade elétrica ao longo do período de cura para as misturas do estudo, misturas 1 a 7. A Figura 5, por sua vez, apresenta os valores da resistividade elétrica em função da idade de cura, e as tendências de crescimento do valor da resistividade com o tempo, para cada tipo de mistura, em particular para as misturas 1 a 5 , as quais apresentam diferentes valores da relação água/cimento. As misturas 6 e 7 apresentam o mesmo valor de a/c que a mistura 1 . O quadro 5 apresenta os coeficientes angulares das retas de tendência da variação do valor da resistividade elétrica com base em crescimento linear, para as misturas 1 a 5 .

\begin{tabular}{|c|c|c|c|c|c|c|c|c|}
\hline \multicolumn{7}{|c|}{ QUADRO 3: Resultados dos ensaios no estado endurecido em ensaios de resistência à } \\
compressão simples \\
\hline
\end{tabular}




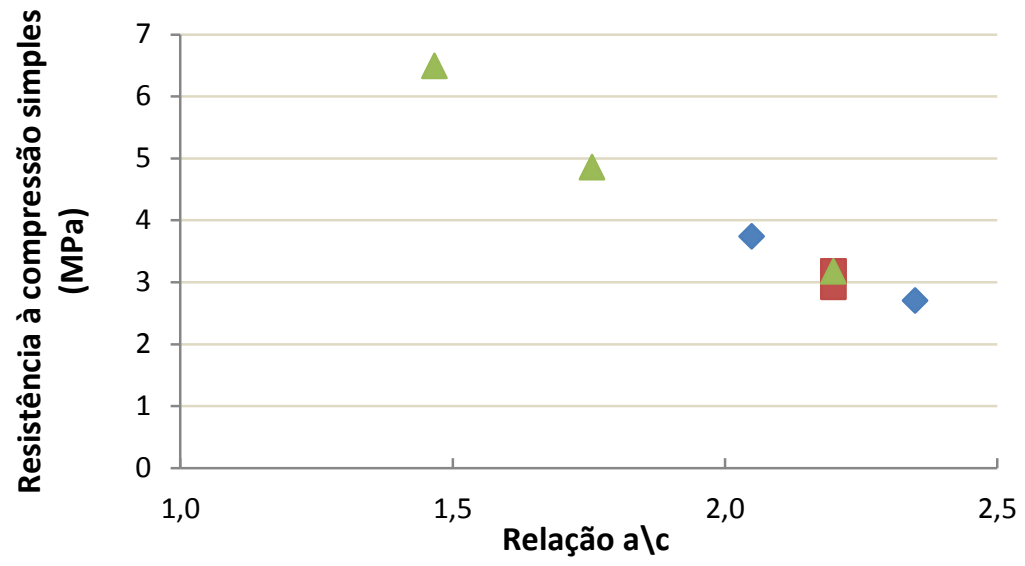

$\diamond$ misturas 1,2 e $3 \square$ Misturas 1,6 e $7 \quad \Delta$ Misturas 1,4 e 5

FIGURA 4: Variação da Resistência à compressão simples em função da relação água/cimento.

\begin{tabular}{|c|c|c|c|c|c|c|c|c|c|}
\hline \multicolumn{7}{|c|}{ QUADRO 4: Resultados da variação da resistividade ao longo do periodo de cura, para as misturas do estudo em } \\
funço dos parâmetros das misturas. \\
\cline { 2 - 10 }
\end{tabular}

QUADRO 5: Coeficientes angulares das retas de tendência linear.

\begin{tabular}{|c|c|c|c|c|c|}
\hline & \multicolumn{5}{|c|}{ Misturas } \\
\hline Mistura & M1 & M2 & M3 & M4 & M5 \\
\hline $\begin{array}{c}\text { Coeficiente } \\
\text { angular da reta } \\
\text { de tendência } \\
\text { linear }\end{array}$ & 0,0228 & 0,0222 & 0,0244 & 0,0263 & 0,0313 \\
\hline
\end{tabular}



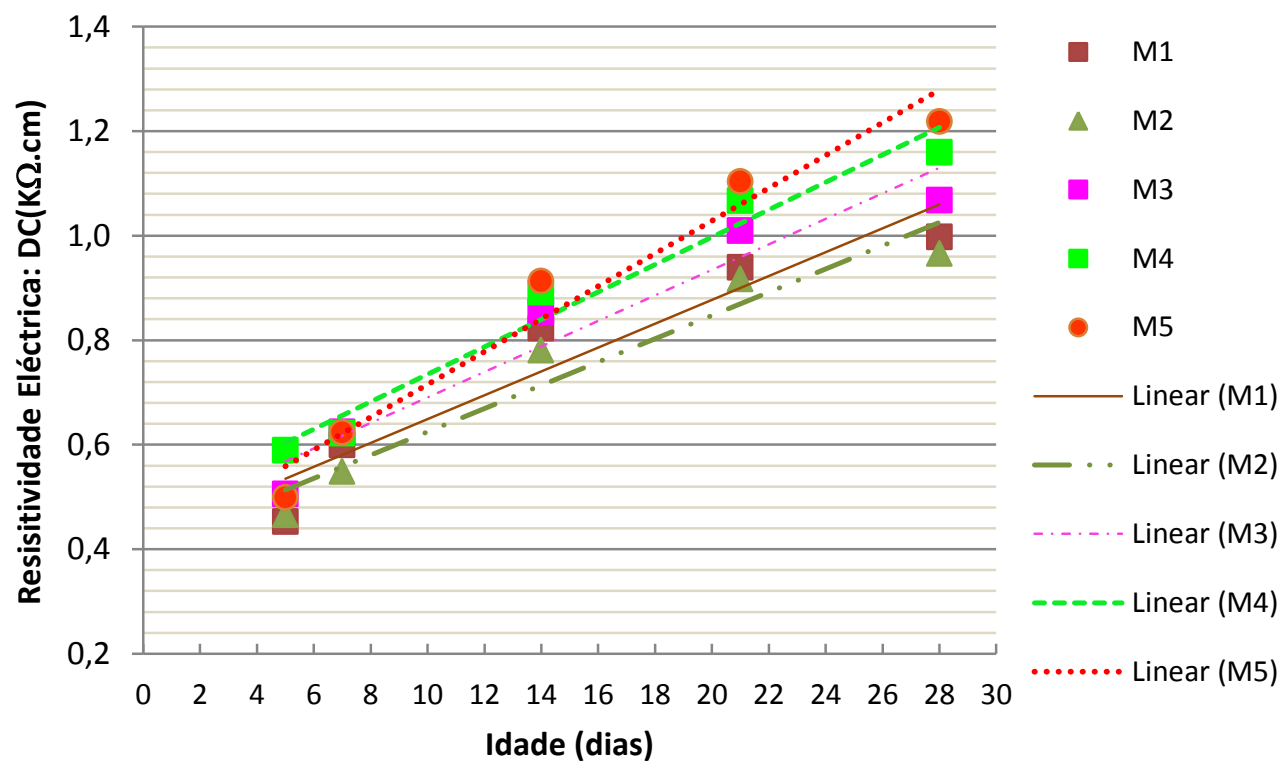

$$
\begin{gathered}
y=0,0228 x+0,4204 \\
R^{2}=0,9089 \\
y=0,0222 x+0,4025 \\
R^{2}=0,935 \\
y=0,0244 x+0,4462 \\
R^{2}=0,9378 \\
y=0,0263 x+0,4724 \\
R^{2}=0,9691 \\
y=0,0313 x+0,4027 \\
R^{2}=0,9618
\end{gathered}
$$

FIGURA 5: Curvas da variação da resistividade referentes às misturas 1 e 5 .

Observa-se pela Figura 5 que o valor da resistividade elétrica aumentou com o tempo de cura para todos os tipos de mistura, dando o indicativo da existência de um processo de hidratação do cimento Portland com a formação de compostos cimentantes, modificando a microestrutura interna e a condição de passagem de corrente elétrica. Isto está coerente com o que é apresentado em Hoppe (2005) para os casos de concretos. Uma análise dos coeficientes angulares das curvas de tendências de crescimento mostrou que a variação de valores da resistividade elétrica é mais acentuada para os casos das misturas com valores menores da relação água/cimento. Neste sentido, a Figura 6 procura ilustrar a correlação possível entre os valores dos coeficientes angulares e a relação água/cimento, com base nas informações dos Quadros 3 e 4.

Nota-se, por meio da Figura 6 , que a variação da resistividade elétrica com o tempo de cura é fortemente influenciada pelo valor da relação água/cimento, coerente com o nível de cimentação. A Figura 7 ilustra a variação do valor da resistividade elétrica aos 28 dias de cura, em função do valor da relação água/cimento e a Figura 8, o valor da resistividade elétrica em função do valor da resistência mecânica alcançada, para as idades de 5 e 28 dias de cura.

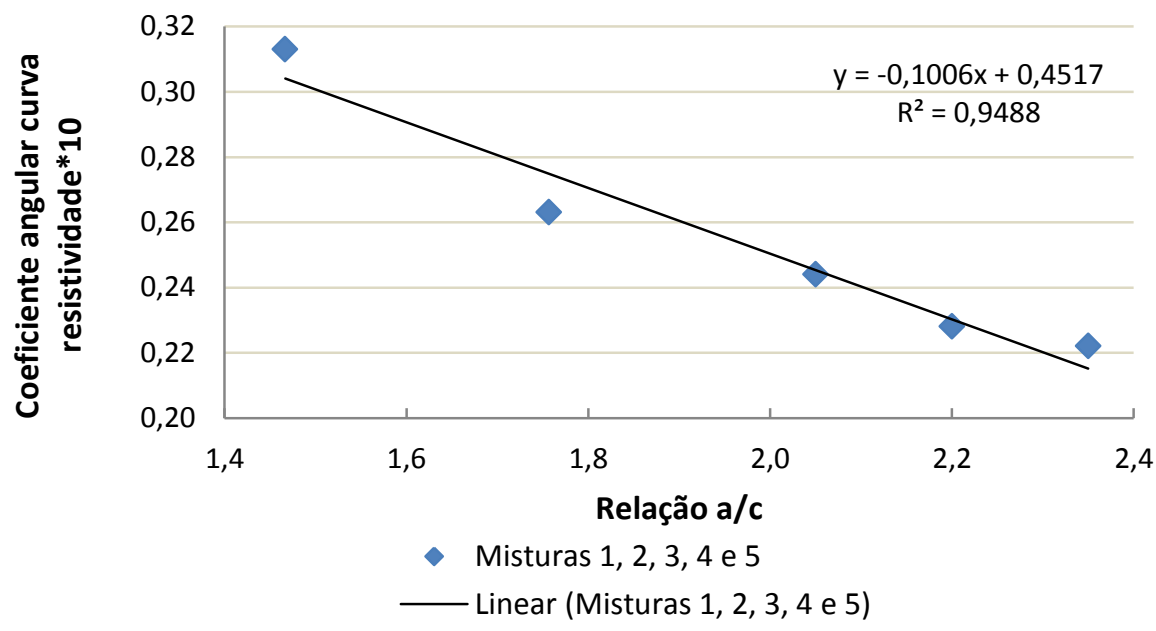

FIGURA 6: Relação água/cimento e o coeficiente angular da curva de resistividade elétrica. 


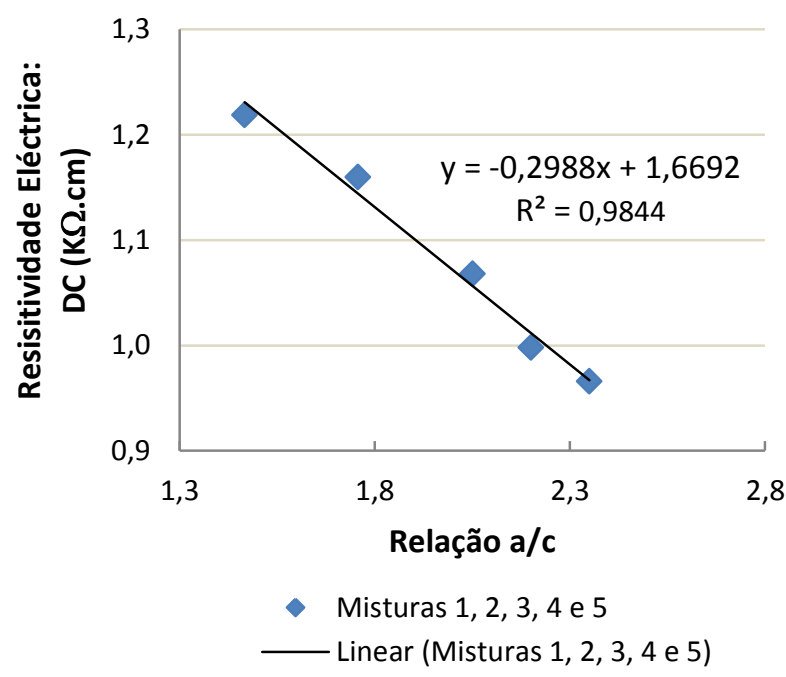

FIGURA 7: Resistividade elétrica aos 28 dias de cura em função da relação água/cimento.

Pela observação da Figura 8, o valor da resistividade elétrica pode ser associado ao valor da resistência mecânica, sendo ele melhor definido para a idade de 28 dias de cura. Com base nas informações dos Quadros 2 e 4, e objetivando obter um modelo de previsão para os valores de resistividade elétrica aos 28 dias de cura com base nas medições dos valores aos 5 dias de cura, o Quadro 6 e a Figura 9 procuram apresentar as

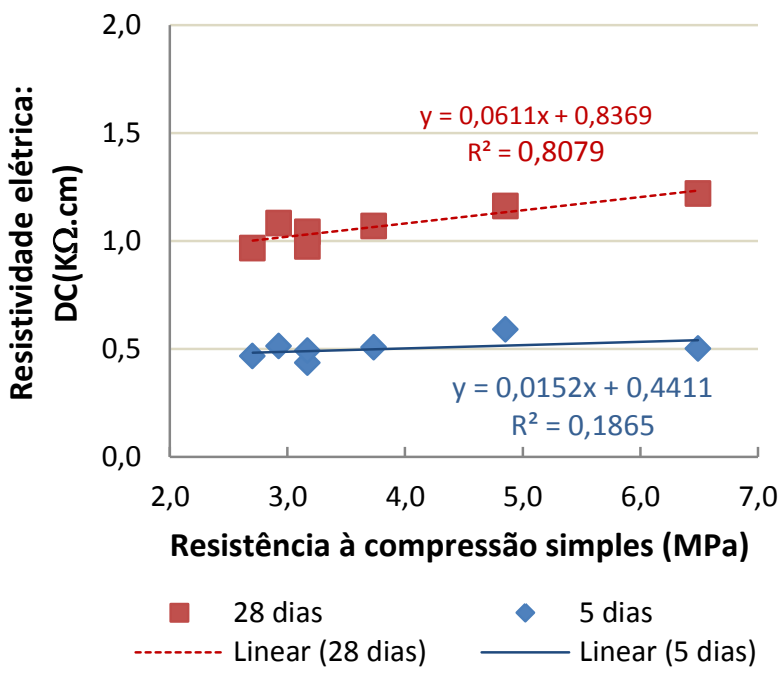

FIGURA 8: Resistividade elétrica aos 28 dias de cura em função da resistência mecânica.

possíveis relações entre estes valores de resistividade aos 5 e aos 28 dias de cura. Conforme ilustra a Figura 9, independentemente do valor da relação água/cimento, o valor da desta relação apresenta pouca variação.

O Quadro 7 e a Figura 10 procuram ilustrar as velocidades do ultrassom para as diversas misturas, para as idades de cura dadas por 5 e 28 dias.

QUADRO 6: Relação entre os valores das resistividades elétricas obtidos para 5 e 28 dias de cura em função do valor da relação água/cimento .

\begin{tabular}{|c|c|c|c|c|c|c|}
\hline & M1 & M2 & M3 & M4 & M5 & \multirow{3}{*}{$\begin{array}{l}\text { Valor médio }=0,531 \\
\text { Desvio padrão }=0,051 \\
\text { Coef. variação }=9,7 \%\end{array}$} \\
\hline Relação a/c & 2,200 & 2,350 & 2,050 & 1,757 & 1,467 & \\
\hline $\begin{array}{l}\text { Resistividade elétrica } 5 \text { dias/ } \\
\text { Resistividade elétrica } 28 \text { dias }\end{array}$ & 0,454 & 0,569 & 0,585 & 0,537 & 0,512 & \\
\hline
\end{tabular}

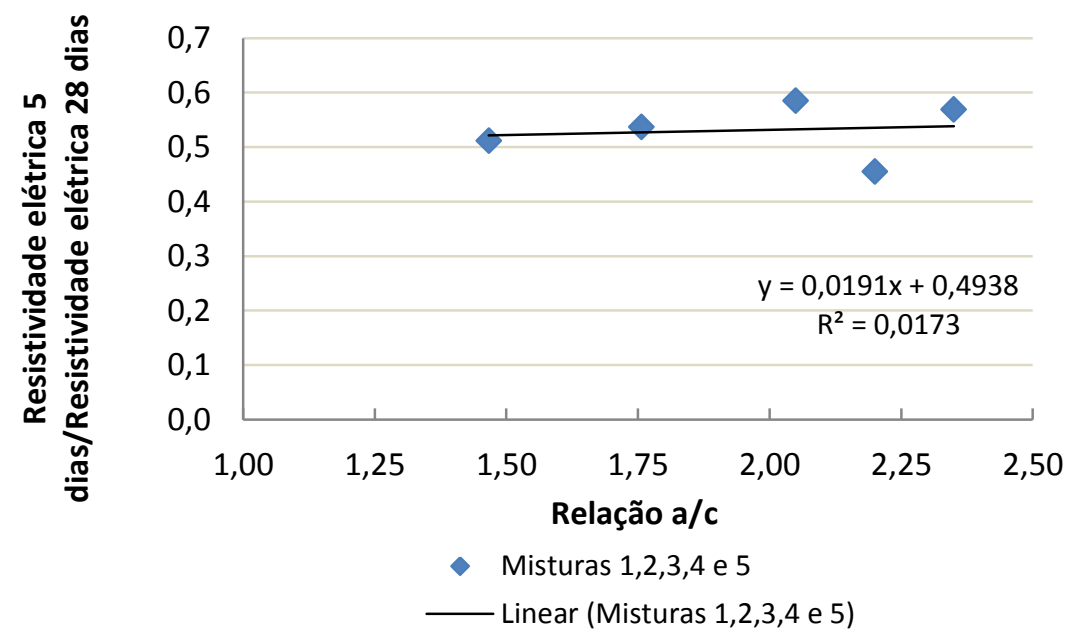

FIGURA 9: Relação entre os valores da resistividade elétrica aos 5 e 28 dias de cura em função da relação água/cimento. 
QUADRO 7: Variação da velocidade de ultrassom para as idades de 5 e 28 dias de cura para as misturas 1 a 7.

\begin{tabular}{|c|c|c|c|c|c|c|c|}
\hline Dias de cura & M1 & M2 & M3 & M4 & M5 & M6 & M7 \\
\hline $\mathbf{5}$ & 1,750 & 1,692 & 1,844 & 1,924 & 2,140 & 1,770 & 1,764 \\
\hline $\mathbf{2 8}$ & 2,251 & 2,236 & 2,368 & 2,493 & 2,686 & 2,280 & 2,220 \\
\hline
\end{tabular}

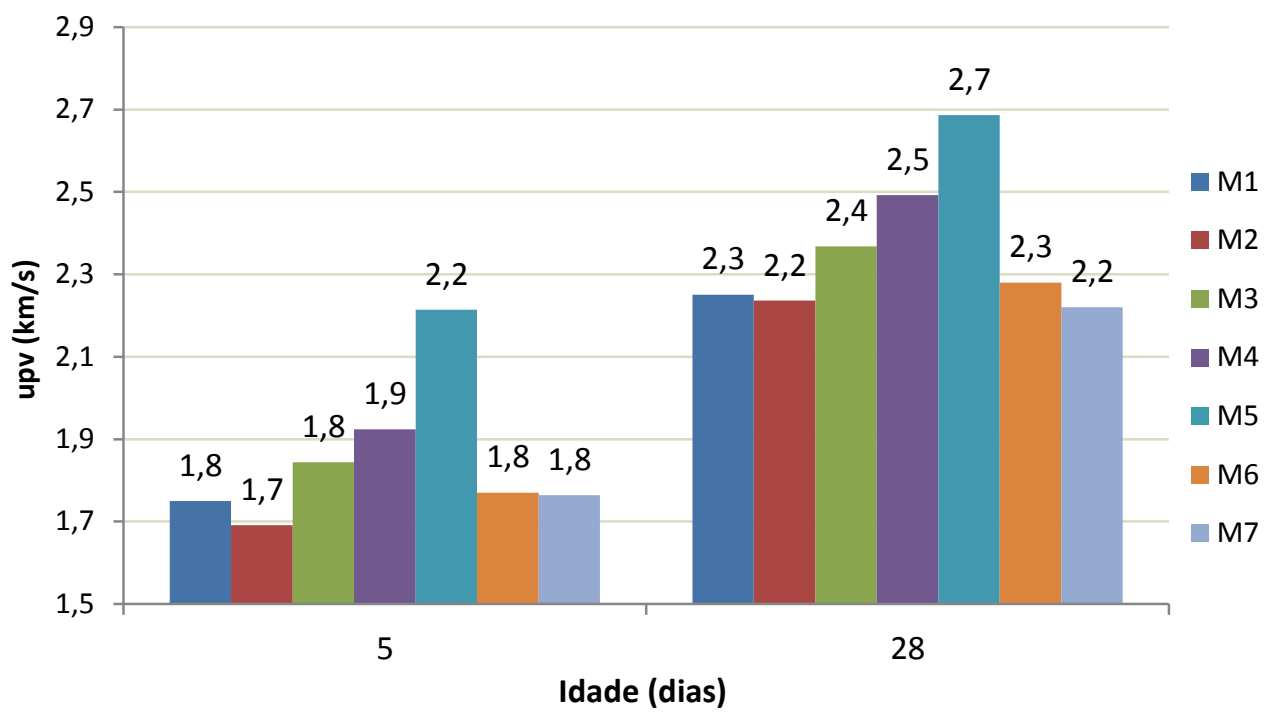

FIGURA 10: Variação da velocidade do ultrassom aos 5 e 28 dias curas para as diversas misturas.

Observa-se do Quadro 7 e da Figura 10 que houve a variação da velocidade das ondas de ultrassom quando consideradas as idades de cura dadas por 5 e 28 dias, variações supostas de que sejam ocorridas em razão da cimentação interna conferida pela presença do cimento. Nota-se também que os valores mais elevados de permissividade de ondas de ultrassom estão associados às misturas que apresentaram os menores valores quanto à relação água/cimento, coerente com os níveis de cimentação que podem ser associados para cada um destes casos. Buscando encontrar para a propagação das ondas de ultrassom um comportamento semelhante ao do caso da variação da resistividade elétrica, com relação ao nível de cimentação, procurou-se encontrar possíveis correlações entre os valores da velocidade de ultrassom com os valores da relação água/cimento, cujos resultados estão ilustrados na Figura 11, para as idades de 5 e de 28 dias. A Figura 12, por sua vez, ilustra a variação da velocidade de ultrassom em função do valor da resistência mecânica alcançada para as idades de cura de 5 e de 28 dias.

Ainda, considerando a possibilidade de se estimar o valor da resistência mecânica aos 28 dias de cura com base nos valores medidos para a velocidade das ondas de ultrassom aos 5 dias, são apresentadas no Quadro 10, as relações entre os valores médios obtidos conforme os valores do Quadro 8, para as idades de 28 e 5 dias, e a Figura 13 apresenta a relação entre estes valores obtidos conforme a variação da relação água/cimento. 


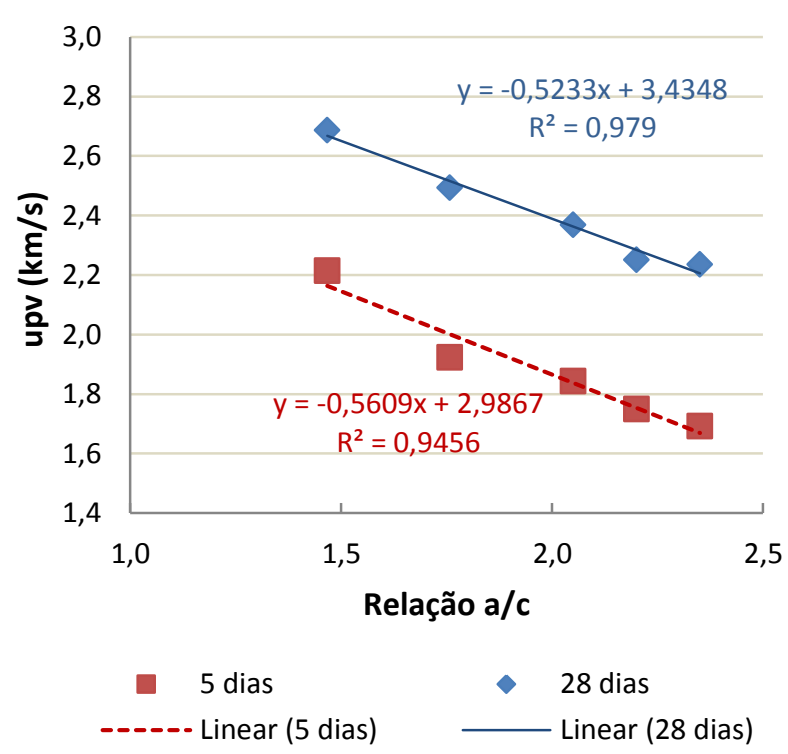

FIGURA 11: Velocidade de propagação de ultrassom aos 5 e 28 dias de cura em função da relação água/cimento.

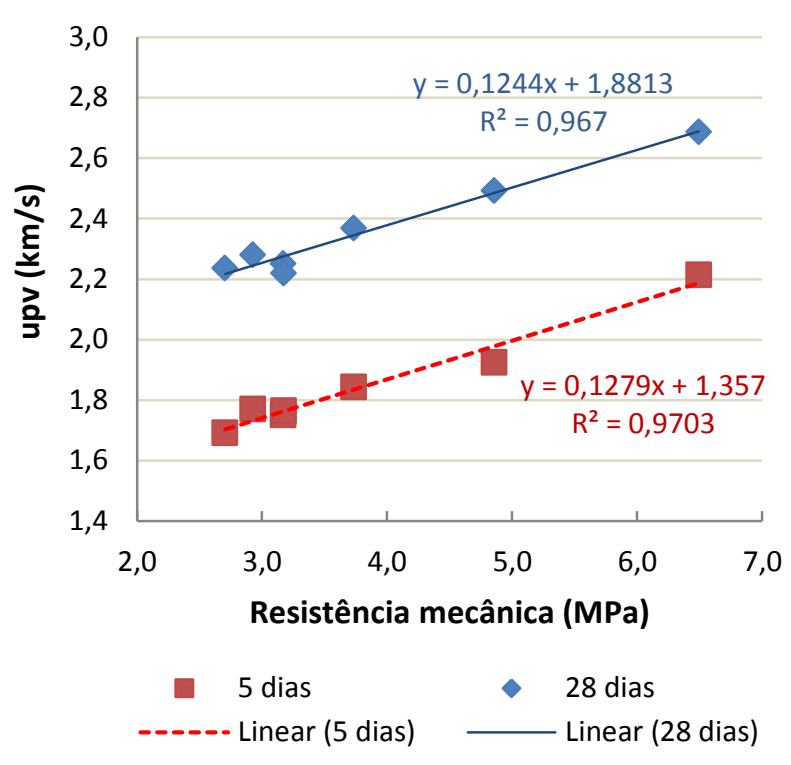

FIGURA 12: Velocidade de propagação de ultrassom aos 5 e 28 dias de cura em função da resistência mecânica.

QUADRO 8: Relação entre valores de UPV para as díades de 5 e 28 dias de cura para as diversas misturas do estudo.

\begin{tabular}{|c|c|c|c|c|c|c|c|}
\hline & $M 1$ & $M 2$ & $M 3$ & $M 4$ & $M 5$ & $M 6$ & M7 \\
\hline $\begin{array}{c}\text { Upv 28 dias } \\
\text { /upv 5 dias }\end{array}$ & 1,286 & 1,322 & 1,284 & 1,296 & 1,213 & 1,288 & 1,258 \\
\cline { 2 - 7 } & \multicolumn{7}{c|}{ Valor médio $=1,278$ Desvio padrão $=0,034 \quad$ Coeficiente variação $=0,027 \%$} \\
\hline
\end{tabular}

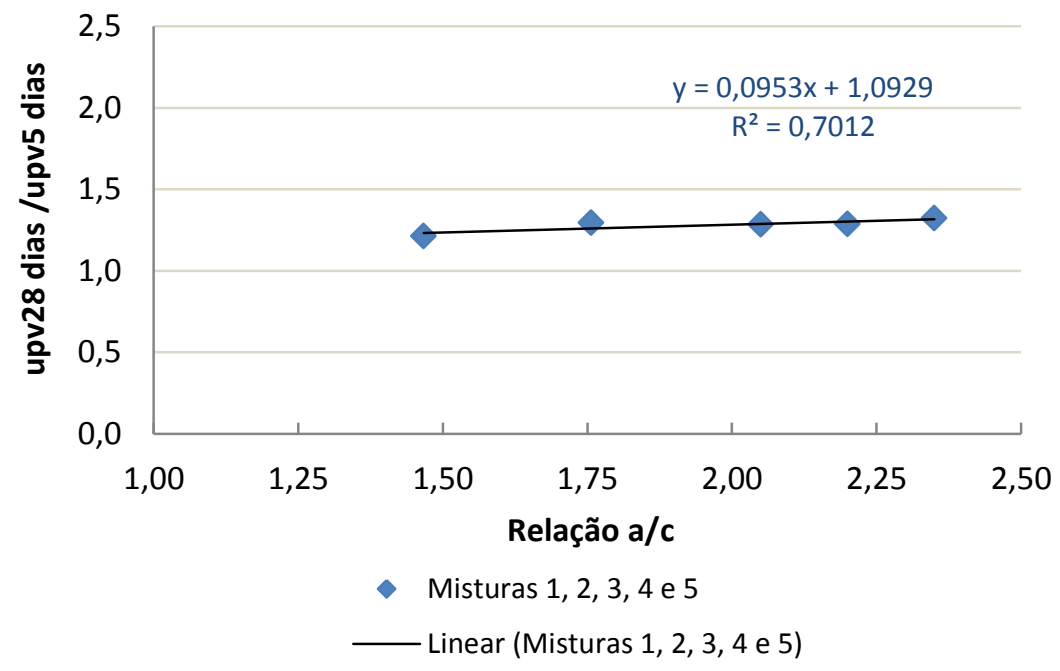

FIGURA 13: Relação entre os valores da velocidade de ultrassom aos 5 e 28 dias de cura em função da relação água/cimento. 
Observa-se que os valores da relação citada tendem a se apresentar pouco variáveis em função do valor da relação água cimento. Convém observar que o valor da relação entre os valores de velocidade das ondas de ultrassom para as idades de 28 dias de cura e 7 dias é dada por 1.278 , valor médio, e próximo ao valor entre as resistências mecânicas alcançadas para a pasta de cimento Portland dadas aos 28 e aos 7 dias de cura, dado por 1,25 conforme a literatura clássica para o caso cimentos Portland (PETRUCCl, 1978).

Considera-se então que houve coerência entre os valores medidos para os ensaios de resistividade elétrica com os de ultrassom, havendo correspondência entre os valores alcançados com as condições de cimentação dos corpos de prova. Neste sentido, buscando uma confirmação na tendência de comportamento, a Figura 14 ilustra a variação dos coeficientes angulares das curvas de tendência de crescimento dos valores de resistividade elétrica, apresentados conforme Quadro 5 e a Figura 5, com os valores da velocidade de ultrassom para 5 e 28 dias de cura, apresentados no Quadro 7. A Figura 15 procura apresentar a correlação entre os valores de resistividade elétrica e a velocidade de ultrassom aos 28 dias de cura. Assim, pode-se observar que os valores de variação da resistividade elétrica e da valocidade de ultrassom se encontram de modo coerente, visto que supostamente estão distribuidos de maneira a confirmar a existência de um processo interno de cimentação, em razão da presença do cimento portland como aditivo.

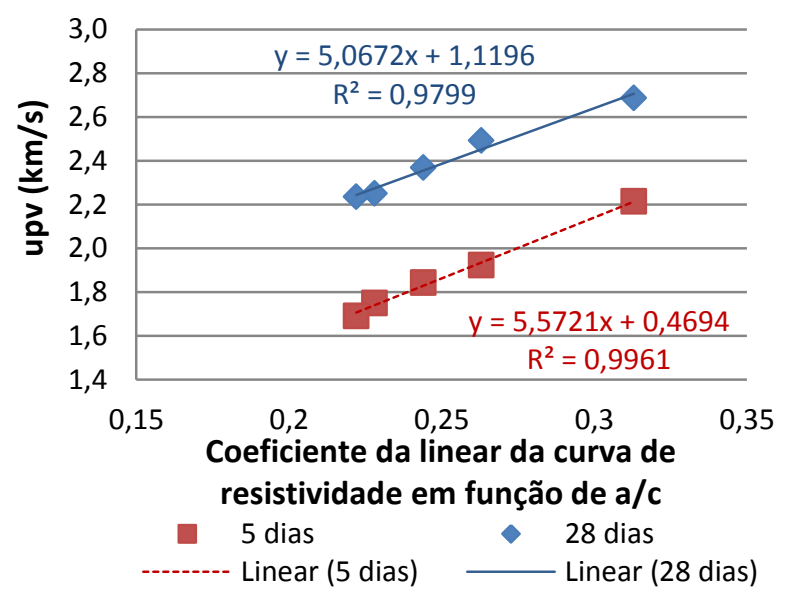

FIGURA 14: Coeficiente de curva de tendencia de crescimento para o caso do valor da resistividade e a velocidade de propagação de ultrassom.

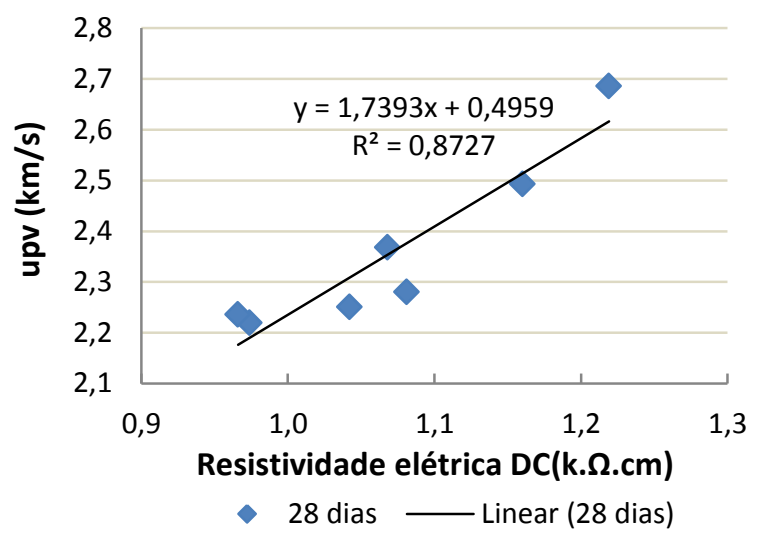

FIGURA 15: Valor da resistividade e a velocidade de propagação de ultrassom e resistividade elétrica.

\section{CONCLUSÕES}

Quanto à evolução da resistência mecânica e as propriedades físicas avalidadas, as avaliações da resistividade elétrica e da velocidade de propagação de ultrassom permitiram indicar a ocorrencia de um processo interno de cimentação, similar aos dos concretos e de argamassas, de modo que, com reações de hidratação se permitem a formação de uma estrutura cimentante, cuja intensidade do processo pode variar em função inversa do crescimento da relação água/cimento. As avaliações da resistividade elétrica e da velocidade de ondas ultrassom se mostraram bastante coerentes entre si, e permitindo estabelecer um modelo previsional para o valor da resistência mecânica.

Diante destes resultados, como sugestão para futuros trabalhos, considera-se relevante um estudo para este tipo de material envolvendo a caracterização da microestrutura formada, de modo a se compreender melhor a evolução e a estruturação do gel.

\section{AGRADECIMENTOS}

Agradecemos à UNESP - Universidade Estadual Paulista, programa de melhoria da pós-graduação, pelo auxílio financeiro para a realização deste estudo, à FEUP - Universidade do Porto, e ao LABEST - Laboratório da Tecnologia do Betão e do Comportamento Estrutural, pelo apoio recebido para a realização da pesquisa. 


\section{REFERÊNCIAS BIBLIOGRÁFICAS}

ALCÂNTARA, M. A. M. Bétons auto-plaçants et fibrages hybrides: composition, rhéologie et comportement mécanique. 2004. 192f. Tese (Doutorado em Engenharia Civil) - Institut National des Sciences Appliquées - INSA, Toulouse, 2004.

ANDRADE, C. Manual para diagnóticos de estruturas deterioradas por corrosão de estruturas. 1992, São Paulo, Pini.

BERTÉ, S. D. D; ALCANTARA, M. A. M. Estudo do comportamento do solo-cimento auto-adensável. REEC - Revista Eletrônica de Engenharia Civil - ISSN 2179-0612, v. 7, n. 2, p. 16-31, 2013.

CONSOLI, N. C.; FOPPA, D.; FESTUGATO, L; HEINECK, K. S. Key Parameters for Strength Control or Artificially Cemented Soils. Journal of Geotechnical and Geoenvironmental Enginnering, New York, v. 133, n. 2, p.197-205, 2007.

FONSECA, A. V; CRUZ, R. C; CONSOLI, N. C. Strength properties of a sandy soil-cement admixtures. Journal Geotechnical \& Geological Engineering, Oklahoma, v. 27, n. 6, p. 681-686. 2009.

HOPPE, T.S. Resistividade elétrica de concretos contendo diferentes teores de cinza de casca de arroz. (Dissertação de mestrado) UFSM - Universidade Federal de Santa Maria - Santa Maria - RS 156p. 2005.

HORPBILSUK, S.; MIURA N.; NAGARAJ, T. S. Assessment of strength development in cement-admixed high water content clays with Abram's law as a basis. Geotechnique Journal, Bangalore, v. 53, n. 4, p. 439-444, 2003.

INSTITUTO PORTUGUÊS DE QUALIDADE - IPQ. Metodo de ensaios de cimentos, parte 1: Determinação das resistências mecânicas. 37p. 1996

MALHOTRA, V. M. \& CARINO, N. J. Handbook on Nondestructive Testing of concrete. CRC Press: Boca Raton, 2004.

NUNES, S. C. B. Betão Auto-Compactável: Tecnologia e Propriedades. Dissertação de Mestrado, Faculdade de Engenharia da Universidade do Porto, Porto, Setembro, 198p. 2001.

PETRUCCI, E.G. Concreto de cimento Portland. Editora Globo, 307p. 1978

ROSA, D.W. Resistividade elétrica de concretos com diferentes teores de escória de alto forno e ativador químico. (Dissertação de mestrado). UFSM Universidade Federal de Santa Maria - Santa Maria - RS 148p. 2005.
SEGANTINI, A. A. S; ALCANTARA, M. A. M. Solo-cimento e solo-cal. In: INSTITUTO BRASILEIRO DE CONCRETO IBRACON (Org.). Materiais de construção civil e princípios básicos de ciência e engenharia de materiais. 2. ed. São Paulo: IBRACON, 2010. v. 2, p. 863-891. 